Aims. The aim of our review was to ensure that:

Curricula are aligned to the GMC's GPC and Excellence by

Design Frameworks

Curricula are capability focused

Curricula promote a flexible and adaptable approach to training

Curricula are succinct, user friendly, patient-centred and reflective of current training in practice

Background. In response to recommendations outlined in the Shape of Training Review (2013), the GMC developed their new framework for postgraduate medical education Excellence by Design (2015), alongside their Generic Professional Capabilities (GPC) Framework (2015).

Method. Governance

To manage the review, a Curriculum Revision Working Group (CRWG) was set up to monitor and govern the review process. Members include Specialty Advisory Committee (SAC) chairs, trainee and patient/lay representatives.

Curriculum Development \& Framework

The CRWG, alongside SACs and specialty working groups, have undertaken a "Why, What, How" approach in developing the curriculum framework. Each curriculum is structured as follows:

High Level Outcomes (HLOs) - These outline the "Why", and provide an overarching view on what should be achieved by trainees. Each HLO is mapped directly onto each of the nine GMC GPC domains.

Key Capabilities - These outline the "What", and provide key detail on what trainees need to undertake to fulfil specific aspects of the curriculum.

Training illustrations - These outline the "How", and supplement the Key Capabilities by providing real-world examples of how to achieve each capability.

Development of the curricula included:

Mapping current Intended Learning Outcomes (ILOs) to the new HLO framework

Re-writing competencies so that they were capability focused

Undertaking a thematic analysis of the curricula, to develop key themes/groupings for capabilities

Review and update Workplace Based Assessments (WBPAs) to ensure they align to the new framework

Stakeholder Engagement

Part of the review has been to ensure Key Stakeholders are involved at each stage of curriculum development. To ensure that all key stakeholders are provided opportunity for consultation, a stakeholder map was developed.

Stakeholder engagement has included:

Direct trainee/trainer/patient/lay involvement at curriculum review meetings

Consultation surveys at each development stage, including feedback on the draft curriculum framework and feedback on full draft curricula

Attendance at meetings with key stakeholders, including NHS Employers and Royal College meetings

Result. The review is currently ongoing. In 2020 we were successful in submitting all 10 of our curricula to the GMC for approval. We are continuing to further develop our curriculum framework, which includes:

Psychiatry "Silver Guide"

Curricula documents

Training illustrations

ARCP Decision Aids

Supplementary Guidance
Conclusion. The review of RCPsych curricula has provided an excellent opportunity to broaden curriculum capabilities, and ensure that the curricula are achievable and deliverable. Our aim is to ensure that the new curricula promote flexibility and adaptability within training, and are user friendly for both trainees and trainers.

\section{Redesigning the psychiatry induction}

Sharadha Wisidagama* and Martin Schmidt

Surrey and Borders Partnership NHS Foundation Trust

${ }^{\star}$ Corresponding author.

doi: 10.1192/bjo.2021.450

Aims. To analyse the current psychiatry induction programme with regards to national guidance, local requirements, trainee and trainer feedback and implement recommendations to streamline where possible.

Background. Junior doctors in training rotate every 4 or 6 months depending on the grade/programme group. GP and FY trainees are often new to psychiatry therefore require a comprehensive induction.

Our Trust has had a three day induction for new junior doctors comprised of 1 day Corporate Induction, 1 day Electronic Records Training and 1 day Local induction.

During the 3 day induction programme there is often a service gap with covering out of hours and acute services. Trainees and trainers have expressed concern regarding the service gap.

We therefore embarked on a review of the induction programme to investigate whether it could be improved in content and length of time to deliver.

Method. Review the regulatory bodies requirements for junior doctor induction.

Gain an understanding of the trainees and trainers perspective of the induction programme.

Review the items in the induction programme according to the requirements of the regulatory bodies.

Tailor the induction programme for junior doctors' needs whilst complying with the regulatory bodies requirements.

Result. The General Medical Council (GMC), British Medical Association (BMA), Gold Guide, Health Education England (HEE) and National Health Service (NHS) employment have no specific statutory and mandatory training requirements for induction.

The regulatory bodies have generic standards for junior doctor induction.

Induction is the responsibility of the Trust.

Trainee perspective: Electronic record system, Mental Health Act (MHA) and pharmacy training were agreed as needing review in terms of its content and length.

Trainees also requested extra items to be included in the induction programme to support successful transition in to their work placements.

The education department met with the Digital Team, MHA Team and Pharmacy Team to develop new and more relevant course content and add in the requested items.

The new induction programme was launched in December 2019 and was reduced in length from 3 to 2 and a half days. Trainee satisfaction improved as evidence by trainee feedback.

Conclusion. The review was helpful in establishing the requirements for a good induction and highlighting areas for improvement.

The new induction was more focussed, shorter in duration and had improved trainee feedback.

The Medical Education Department will assess the changes following the December 2019 induction and continue to review its induction programme. 